\title{
Highly Efficient Hierarchical Micelles Integrating Photothermal Therapy and Singlet Oxygen-Synergized Chemotherapy for Cancer Eradication
}

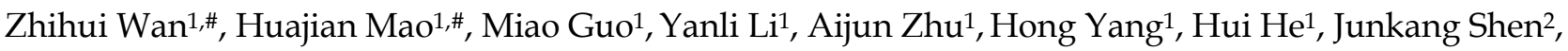 \\ Lijuan Zhou' ${ }^{2}$, Zhen Jiang'2, Cuicui Ge3 ${ }^{3}$ Xiaoyuan Chen', Xiangliang Yang5, Gang Liu', and Huabing \\ Chen $1,3, \square$ \\ 1. Jiangsu Key Laboratory of Translational Research and Therapy for Neuro-Psycho-Diseases, and College of Pharmaceutical Sciences, \\ Soochow University, Suzhou 215123, China \\ 2. Radiology Department, Second Affiliated Hospital, Soochow University, Suzhou 215004, China \\ 3. School for Radiological \& Interdisciplinary sciences (RAD-X), and School of Radiation Medicine and Protection, Soochow University, \\ Suzhou 215123, China \\ 4. Laboratory of Molecular Imaging and Nanomedicine (LOMIN), National Institute of Biomedical Imaging and Bioengineering (NIBIB), \\ National Institutes of Health Bethesda, Maryland 20892, United States \\ 5. College of Life Science and Technology, Huazhong University of Science and Technology, Wuhan 430074, China \\ 6. State Key Laboratory of Molecular Vaccinology and Molecular Diagnostics \& Center for Molecular Imaging and Translational Medicine, \\ School of Public Health, Xiamen University, Xiamen 361102, China
}

\# These authors contributed equally

$\triangle$ Corresponding author: E-mail: chenhb@suda.edu.cn

() Ivyspring International Publisher. This is an open-access article distributed under the terms of the Creative Commons License (http://creativecommons.org/ licenses/by-nc-nd/3.0/). Reproduction is permitted for personal, noncommercial use, provided that the article is in whole, unmodified, and properly cited.

Received: 2013.1I.19; Accepted: 2014.01.06; Published: 2014.01.29

\begin{abstract}
It is highly desirable to develop theranostic nanoparticles for achieving cancer imaging with enhanced contrast and simultaneously multimodal synergistic therapy. Herein, we report a theranostic micelle system hierarchically assembling cyanine dye (indocyanine green) and chemotherapeutic compound (doxorubicin) (I/D-Micelles) as a novel theranostic platform with high drug loading, good stability and enhanced cellular uptake via clathrin-mediated endocytosis. I/D-Micelles exhibit the multiple functionalities including near-infrared fluorescence (NIRF), hyperthermia and intracellular singlet oxygen from indocyanine green, and simultaneous cytotoxicity from doxorubicin. Upon photoirradiation, I/D-Micelles can induce NIRF imaging, acute photothermal therapy via hyperthermia and simultaneous synergistic chemotherapy via singlet oxygen-triggered disruption of lysosomal membranes, eventually leading to enhanced NIRF imaging and superior tumor eradication without any re-growth. Our results suggest that the hierarchical micelles can act as a superior theranostic platform for cancer imaging and multimodal synergistic therapy.
\end{abstract}

Key words: Hierarchical micelles; Photothermal therapy; Singlet oxygen; Synergistic therapy; Cancer eradication

\section{Introduction}

Photothermal therapy (PTT) has been considered as a minimally invasive means of cancer therapy, which generally employs photo-absorbing agents to generate heat under photoirradiation [1-3]. In the past decades, several types of agents with strong near-infrared (NIR) absorbance have been explored as 
the photo-absorbing candidates for PTT, such as cyanine dyes, gold nanoparticles as well as graphene oxide [1, 4-6]. Generally, PTT often requires sufficient tumor accumulation of these photo-absorbing agents to reach sufficient hyperthermia $\left(>42{ }^{\circ} \mathrm{C}\right)$ for triggering photothermal effect after imaging-guided tumor identification and localization. However, even though PTT is highly effective to inhibit tumor growth, it is possible to cause residual cells surviving from photothermal injury and subsequent tumor re-growth upon insufficient tumor accumulation of the photo-absorbing agents $[4,5,7]$. Consequently, it is still a major challenge to achieve tumor eradication without tumor re-growth using PTT in the field of cancer therapy.

Recently, PTT-based multimodal therapy has been found to provide preferable efficacy than respective monotherapy, implying that multimodal therapy may achieve better therapeutic efficacy [5, 7-10]. Ideal multimodal therapy such as the combination of PTT and chemotherapy is expected to generate not only combinational effect, but also synergistic effect to achieve unprecedented therapeutic outcome such as the eradication of residual cells surviving from PTT. So far, there are only a few investigations on intracellular synergism between various anticancer agents owing to the absence of the synergistic mechanism $[4,11]$. It is thus intriguing to explore a new synergistic mechanism of PTT-based multimodal therapy.

Cyanine dyes possess promising photothermal effect without safety concern compared to inorganic photo-absorbing nanomaterials [3]. In particular, cy- anine dyes such as indocyanine green (ICG) as clinically potential agents display multifunctional properties including fluorescent emission of above $800 \mathrm{~nm}$ and generation of singlet oxygen in addition to photothermal effect [3, 12-17]. NIR fluorescence (NIRF) of cyanine dyes can offer a potential capacity to achieve cancer imaging with minimal auto-fluorescence interference [3], which can provide ultrasensitive cancer detection and allow us to precisely photo-irradiate tumor for effective tumor growth inhibition as shown in our previous studies [17]. On the other hand, singlet oxygen is considered as a highly reactive oxygen species (ROS) in cells, which plays an important role in the damage of subcellular organelles [18]. However, the role of singlet oxygen from cyanine dyes such as ICG in intracellular delivery of anticancer agent is ignored, although its photodynamic effect has been evaluated [13]. Herein, we report a hierarchical micelle system assembling cyanine dye (ICG) and chemotherapeutic agent (doxorubicin, DOX) (I/D-Micelles) as a highly efficient platform integrating PTT and singlet oxygen-synergized chemotherapy for cancer eradication (Scheme 1/Figure A). Upon photoirradiation, I/D-Micelles can induce a concentration-dependent hyperthermia and simultaneous generation of singlet oxygen at a relatively low concentration range. In particular, we for the first time demonstrated that singlet oxygen from permeable hierarchical micelles is able to trigger the destabilization of lysosomes upon photoirradiation, which maximize the accessibility of DOX to nucleus and subsequently synergize the cytotoxicity of DOX with PTT for cancer eradication.

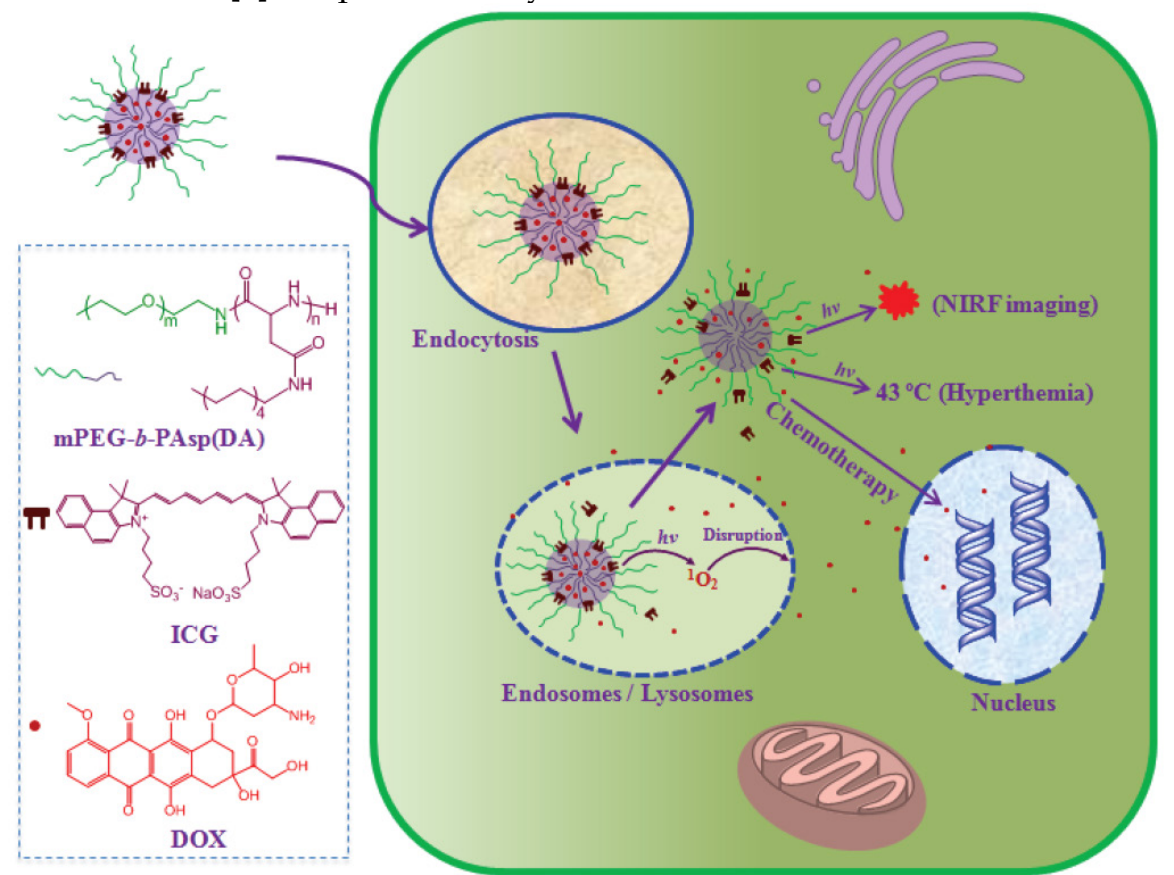

Figure A. (Scheme I) Schematic illustration of highly efficient hierarchical cyanine-based micelles integrating photothermal therapy and synergistic chemotherapy for cancer eradication. 


\section{Materials and methods}

\section{Materials}

$\beta$-benzyl-L-aspartate N-carboxyanhydride (BLANCA) was obtained from Enlai Biological Technology Company (Chengdu, China). mPEG-NH 2 (12000 MW) was purchased from Jenkem Technology Company (Beijing, China). mPEG-b-PAsp was synthesized as mentioned method in the reference (The degree of polymerization of PAsp segment was calculated to be 56 according to ${ }^{1} \mathrm{H}-\mathrm{NMR}$ spectrum, Supplementary Material: Fig. S1) [17, 19]. AO was purchased from Amresco. N-Methyl pyrrolidone (NMP), ICG and decylamine were obtained from Sigma-Aldrich. DHE was obtained from Wusheng Company (Shanghai, China).

\section{Synthesis and preparation}

mPEG- $b$-PAsp was reacted with decylamine in $\mathrm{NMP}$ at $45{ }^{\circ} \mathrm{C}$ overnight. Then, the solutions were dialyzed in the mixture of ethanol and water for removing excess amines and then were dialyzed in water. Finally, mPEG- $b$-PAsp(DA) was obtained by lyophilizing the dialyzed solutions, and their degrees of polymerization were calculated to be 52 according to ${ }^{1} \mathrm{H}-\mathrm{NMR}$ spectrum (Supplementary Material: Fig. S2).

$2 \mathrm{mg}$ ICG, $2 \mathrm{mg}$ DOX and $6 \mathrm{mg}$ mPEG- $b$ PAsp(DA) were mixed at the ratio of 1:1:3 in the mixture $(5 \mathrm{ml})$ of $\mathrm{CH}_{2} \mathrm{Cl}_{2}$ and triethylamine, and then the solutions were evaporated using rotary evaporator. Subsequently, the micelles were prepared by dispersing the evaporated mixture in de-ionized water and purified using centrifuge separation via ultrafiltration (Cut-off $100 \mathrm{~K} \mathrm{MW}$ ) at $4{ }^{\circ} \mathrm{C}$. ICG-Micelles or DOX-Micelles were prepared by mixing mPEG- $b$ PAsp(DA) with ICG or DOX at the ratio of $4: 1$ according to same procedure, respectively. Free ICG/DOX was obtained by mixing ICG and DOX in PBS.

\section{Characterization}

Dynamic light scattering (Malvern, UK) was used to measure the hydrodynamic diameter of micelles at $25^{\circ} \mathrm{C}$. The absorbance and fluorescent spectrums of ICG or DOX were measured using UV-Vis Spectrophotometer (UV2600, Shimadzu) and Fluorescence Spectrophotometer (LS 55, Perkin Elmer), respectively.

\section{Drug release}

The drug release behaviors of ICG and DOX from I/D-Micelles containing $0.3 \mathrm{mg}$ ICG and $0.3 \mathrm{mg}$ DOX were evaluated using dialysis method respectively, and free ICG/DOX in DMSOwas used as the control. The drug release was performed in air Con- stant Temperature Oscillator shaker at $37^{\circ} \mathrm{C} .100 \mathrm{ml}$ PBS (0.2 M, pH 7.4) was used as the release medium, respectively. The samples $(1.0 \mathrm{~mL})$ were taken after $0.16,0.3,0.5,1,2,4,6,8,10$ and $24 \mathrm{~h}$ with the replacement of an equal volume of fresh medium. UV-Vis spectrometer and Fluorescence Spectrophotometer were used to measure the concentrations of ICG and DOX in the samples, respectively.

\section{Photothermal effect}

I/D-Micelles and free ICG/DOX (each $0.5 \mathrm{~mL}$ ) at the concentrations of $1,2,5,10,25,50,75$ and 100 $\mathrm{\mu g} / \mathrm{mL}$ ICG were stored in the transparent plastic vials and then were irradiated at the excitation wavelength of $785 \mathrm{~nm}\left(1.5 \mathrm{~W} / \mathrm{cm}^{2}\right.$, FS-Optics, China). Simultaneously, the temperature of the solution was monitored during $200 \mathrm{~s}$. PBS was used as the negative control in this experiment.

\section{Cellular uptake}

A549 cells were seeded on 24-well culture plates $\left(3 \times 10^{5}\right.$ cells/well) and incubated overnight in DMEM containing 10\% FBS. I/D-Micelles and free ICG/DOX $(4 \mu \mathrm{g} / \mathrm{mL}$ ICG) were respectively applied to the wells. After 6 and $24 \mathrm{~h}$ incubation, the cells were washed 3 times using PBS. The cells were incubated with $0.5 \mathrm{~mL}$ trypsin (Sigma-Aldrich, USA) for $3 \mathrm{~min}$ at $37^{\circ} \mathrm{C}$, and then the cells were counted after centrifuge collection. The counted cells suffered from ultrasonication, and then ICG and DOX were further extracted from the cells using methanol. Finally, the concentrations of ICG and DOX were measured via UV-Vis and fluorescent assay, respectively. The results are presented as a mean and standard deviation obtained from 6 samples.

\section{Flow cytometry}

A549 cells $\left(1.2 \times 10^{6}\right.$ cells/well $)$ were seeded in 6-well plates. A549 cells were treated with PBS (Control) and the different inhibitors including chlorpromazine (inhibitor of clathrin-mediated uptake, 10 $\mu \mathrm{g} / \mathrm{mL}$ ), filipin (inhibitor of caveolae-mediated uptake, $5 \mu \mathrm{g} / \mathrm{mL}$ ), and amiloride (inhibitor of macropinocytosis, $100 \mu \mathrm{g} / \mathrm{mL}$ ) in serum-free DMEM medium for $1 \mathrm{~h}$ at $37^{\circ} \mathrm{C}$ or $4{ }^{\circ} \mathrm{C}$, respectively. Then, I/D-Micelles (4 $\mathrm{\mu g} / \mathrm{mL}$ DOX/ICG) were further added to the medium for another $1 \mathrm{~h}$ incubation. Subsequently, the cells were washed 3 times using PBS, treated with trypsin, centrifuged at $4{ }^{\circ} \mathrm{C}$, and finally suspended in $0.5 \mathrm{~mL}$ of PBS. The fluorescent intensity of DOX in cells was analyzed by flow cytometry (BD LSR II, NJ) after filtration.

\section{Disruption of lysosomal membranes}

To evaluate the disruption of lysosomal membranes, $\mathrm{AO}$ was utilized as an intracellular indicator 
of acidic organelle integrity in A549 cells [17]. AO is a metachromatic fluorophore that becomes charged and is retained by proton trapping within acidic compartments. When normal cells were excited with blue light, $\mathrm{AO}$ in lysosomes emits an intense red fluorescence, while AO in nuclei and cytosol displays green fluorescence. When acidic compartments such as lysosomes is disrupted, the red fluorescence from $\mathrm{AO}$ disappears and only green fluorescence can be observed. In this study, A549 cells were seeded overnight on glass slides in 35-mm dishes, and treated with PBS and I/D-Micelles at the concentrations of 0.1 and $0.2 \mu \mathrm{g} / \mathrm{mL}$ ICG for $6 \mathrm{~h}$, respectively. Then, the cells were incubated in fresh medium, followed by 3 min photoirradiation at $1.5 \mathrm{~W} / \mathrm{cm}^{2}$. After $1 \mathrm{~h}$, the cells were washed using PBS and fixed for 10 min using 1 $\mathrm{mL}$ mixture of methanol/ glacial acetic acid (3:1). Then, A549 cells were washed using PBS and further incubated with $6 \mu \mathrm{M}$ AO $(1.0 \mathrm{~mL})$ for $15 \mathrm{~min}$. The cells were washed 3 times with PBS before subjected to confocal microscopy imaging (Nikon Eclipse TE2000-E) with the excitation wavelength of $488 \mathrm{~nm}$, and emission wavelengths from 515 to $545 \mathrm{~nm}$ (green) and from 610 to $640 \mathrm{~nm}$ (red).

\section{MTT assay}

A549 cells were incubated with different formulations including free ICG/DOX, ICG-Micelles, DOX-Micelles and I/D-Micelles at various concentrations including $0.005,0.12,0.25,0.5,1.0,2.0$, and 4.0 $\mathrm{\mu g} / \mathrm{mL}$ with or without photoirradiation $\left(1.5 \mathrm{~W} / \mathrm{cm}^{2}\right)$ for $24 \mathrm{~h}$, and then the cells were washed using PBS. After $24 \mathrm{~h}$, the cell viability was evaluated using MTT assay. For photothermal injury, the cells were incubated with ICG-Micelles containing various concentrations of ICG for $24 \mathrm{~h}$, and then suffered from $3 \mathrm{~min}$ photoirradiation at $1.0,1.5$ and $3.0 \mathrm{~W} / \mathrm{cm}^{2}$. Subsequently, the cell viability was measured using MTT assay.

\section{Trypan blue staining}

To validate cell injury, A549 cells were seeded overnight in 24-well plates, and then treated with PBS and I/D-Micelles at the concentration of $4.0 \mu \mathrm{g} / \mathrm{mL}$ ICG/DOX for $24 \mathrm{~h}$, respectively. Then, the cells were incubated with fresh medium and then exposed to a $785 \mathrm{~nm}$ laser at $1.5 \mathrm{~W} / \mathrm{cm}^{2}$. After $1 \mathrm{~h}$, the cells were washed 3 times using PBS, followed by the addition of $0.2 \mathrm{~mL}$ trypan blue solution (0.1 wt \%). Then, A549 cells were observed using an optical microscope after wash. Trypan blue dye molecules can easily penetrate plasma membranes of dead cells to stain the nuclei, but viable cells can not be stained.

\section{Monitoring of singlet oxygen}

Free ICG/DOX and I/D-Micelles containing various concentrations of ICG in PBS solutions containing $32.0 \mu \mathrm{g} / \mathrm{mL}$ DPBF $(1.0 \mathrm{~mL})$ were irradiated for $3 \mathrm{~min}$ at $1.5 \mathrm{~W} / \mathrm{cm}^{2}$ for singlet oxygen measurement. The fluorescent spectrum was scanned from 450 to $565 \mathrm{~nm}$ using an excitation wavelength of $403 \mathrm{~nm}$. Then, the fluorescence intensity at $485 \mathrm{~nm}$ was recorded. The solution of DPBF without ICG in darkness was used as the control.

\section{DHE staining}

Reactive oxygen species (ROS) formation was monitored by fluorescence microscopy using dihydroethidium (DHE) as the indicator. Briefly, cells were seeded on 24-well plates at the density of $2 \times 10^{5}$ each well and incubated with I/D-Micelles containing $0.1,0.2$ and $0.5 \mu \mathrm{g} / \mathrm{mL}$ ICG for $6 \mathrm{~h}$. After the cells were washed 3 times using PBS, $0.2 \mathrm{~mL}$ DHE solution (5 $\mu \mathrm{M}, \mathrm{PBS})$ was added into the cells for $30 \mathrm{~min}$ at $37^{\circ} \mathrm{C}$, simultaneously accompanying with or without $3 \mathrm{~min}$ photoirradiation $\left(785 \mathrm{~nm}, 1.5 \mathrm{~W} / \mathrm{cm}^{2}\right)$. Then, the cells were washed twice using PBS, and observed using an optical microscope with the excitation of green light.

\section{Hemolysis}

Rabbit blood was collected in a heparin-containing tube and centrifuged at $1500 \mathrm{rpm}$ for 5 min to separate red blood cells (RBC) from plasma, which were washed and diluted with isotonic PBS at $\mathrm{pH}$ 7.4. The solutions of copolymer were incubated with $2 \%(\mathrm{v} / \mathrm{v}) \mathrm{RBC}$ suspensions at $37^{\circ} \mathrm{C}$ for $1 \mathrm{~h}$ at 0.4 , $0.8,1.2,1.6$, and $2.0 \mathrm{mg} / \mathrm{mL}$, respectively. The unlysed $\mathrm{RBC}$ were removed by centrifugation, and the supernatant was analyzed by UV/Vis spectroscopy at 545 $\mathrm{nm}$. The percentage hemolysis was determined using the following equation:

$$
\text { Hemolysis }(\%)=\left(\mathrm{A}_{\text {sample }}-\mathrm{A}_{0 \%}\right) /\left(\mathrm{A}_{100 \%}-\mathrm{A}_{0 \%}\right) \times 100 \%
$$

where $\mathrm{A}_{\text {sample, }}, \mathrm{A}_{0 \%}$ and $\mathrm{A}_{100 \%}$ represent the absorbance of the samples, the negative control (PBS) and the positive control (distilled water), respectively.

\section{Biodistribution}

A549 cells $\left(1 \times 10^{7}\right.$ cells/each mouse) were subcutaneously injected into the flanks of female BALB/c nude mice (16-18 g) for constructing the tumor-bearing mice. Then, the tumor-bearing mice were injected intravenously with free ICG/DOX and I/D-Micelles at the dose of $7.5 \mathrm{mg} / \mathrm{kg}$ ICG/DOX, respectively. Then, the various tissues including heart, liver, spleen, lung, kidney and tumor were extracted from the mice at $24 \mathrm{~h}$ post-injection, respectively. Finally, the tissues were imaged using IVIS Lumina II with the excitation wavelength of $745 \mathrm{~nm}$. The NIRF signals from I/D-Micelles in the tissues were calculated to describe the ex vivo biodistribution of ICG at $24 \mathrm{~h}$. Subsequently, the tissues were further 
homogenized in $1.0 \mathrm{~mL}$ physiological saline for measuring the biodistribution of DOX. Methanol and chloroform were respectively used to extract DOX from the solutions. Finally, the samples were re-dissolved using methanol for HPLC analysis of DOX at the wavelength of $254 \mathrm{~nm}$.

\section{In vivo imaging}

A549 cells $\left(1 \times 10^{7}\right.$ cells/each mouse $)$ were subcutaneously injected into the flanks of female BALB/c nude mice (16-18 g) for constructing the mice bearing A549 tumors. Free ICG/DOX and I/D-Micelles were intravenously into the above mice at the dose of 7.5 $\mathrm{mg} / \mathrm{kg}$ ICG or DOX, respectively. Then, the mice were imaging using IVIS Lumina II with the excitation wavelength of $745 \mathrm{~nm}$ at 6, 24, 48, 96, and $144 \mathrm{~h}$ post-injection. The average NIRF intensity at tumor was calculated to describe the photon signals at tumor at different time.

\section{In Vivo efficacy}

A549 cells $\left(1 \times 10^{7}\right.$ cells/each mouse) were subcutaneously transplanted into the flanks of female mice [17]. When the tumors reached a size of $50 \sim 70 \mathrm{~mm}^{3}$ (about 10 days after transplantation), various formulations including PBS, free ICG/DOX, ICG-Micelles, DOX-Micelles, and I/D-Micelles were injected intravenously into the mice at the dose of $7.5 \mathrm{mg} / \mathrm{kg}$ ICG/DOX on day 0,2 , and 4 , respectively. Subsequently, the tumors suffered from 5 min photoirradiation $\left(1.0 \mathrm{~W} / \mathrm{cm}^{2}\right)$ or not at the wavelength of $785 \mathrm{~nm}$ at $24 \mathrm{~h}$ post-injection. The tumor volumes were measured to evaluate the anticancer efficacy of various groups. The tumor volume $(\mathrm{V})$ was calculated as follows: $V=L \times W^{2} / 2$, where $W$ is the tumor measurement at the widest point, and $\mathrm{L}$ is the tumor dimension at the longest point. The tumor volumes are normalized against the original volumes at 0 day for monitoring the tumor growth. The mice were sacrificed by cervical dislocation under an anesthetic status after the experiments ( 26 days post-injection). The statistic difference was analyzed using $t$ test where $P$ value of $<0.05$ is considered significant.

\section{Results and discussion}

\section{Synthesis, preparation and characterization}

To date, there are still some major drawbacks of cyanine dyes (e.g. ICG) for NIRF imaging and PTT including insufficient tumor accumulation and cell internalization, and quick elimination at tumor, which can cause insufficient imaging contrast and poor photothermal efficacy [13, 20-22]. Even though some nanocarriers such as polymeric nanoparticles and calcium phosphate nanoparticles have already been employed to incorporate cyanine dyes (e.g. ICG) to improve the photostability and target specificity for cancer NIRF imaging [3, 20, 22-24], most reports dealt with in vitro photothermal efficiency or in vivo efficacy via intratumor administration, rather than intravenous administration $[7,12,14,21]$. Consequently, the nanostructure with superior cell internalization, enhanced tumor accumulation and retention capabilities is highly expected to provide an ideal strategy to achieve efficient co-delivery of ICG and DOX [25-27]. Polymeric micelles as clinically potential nanocarrier possess multiple advantages such as high loading capacity, good stability, sustained release, prolonged circulation, enhanced permeability and retention (EPR) effect and preferable cell internalization [28-32]. Here, the copolymer consisting of monomethoxy poly(ethylene glycol) and decylamine-grafted poly(l-aspartic acid) (mPEG-b-PAsp(DA)) was synthesized by ring-opening polymerization of $\beta$-benzyl L-aspartate $N$-carboxy-anhydride (BLA-NCA) with mPEG (12 kDa) and subsequent aminolysis of decylamine (Fig. 1) [19, 33]. ${ }^{1} \mathrm{H}-\mathrm{NMR}$ analysis confirmed the chemical structure of the copolymers (Supplementary Material: Fig. S1 and S2) and gel permeation chromatography measurement also confirmed this synthesis $\left(M_{w} / M_{n}=1.08\right.$, data not shown) [17]. In order to evaluate the potential toxicity of mPEG- $b$-PAsp(DA), we evaluated the hemolysis of mPEG- $b$-PAsp(DA) by incubating with red blood cells for $1 \mathrm{~h}$ at different concentrations. The hemolytic percentage of mPEG- $b$-PAsp(DA) was lower than 5\% at the concentration of up to $1.6 \mathrm{mg} / \mathrm{mL}$ ( $5 \%$ is considered as the threshold), indicating that this copolymer has a negligible hemolytic activity (Supplementary Material: Fig. S3).

mPEG- $b$-PAsp(DA), ICG and DOX at the ratio of 3:1:1 were dissolved in the mixture of methanol and triethylamine. Then, the solution was evaporated into an uniform membrane, which was further dispersed into water and purified for the construction of ICG/DOX-loaded mPEG- $b$-PAsp(DA) micelles (I/D-Micelles). I/D-Micelles had the spherical morphology and average diameter of $71.4 \mathrm{~nm}$ with the polydispersity index of 0.2 (Supplementary Material: Fig. S4). We evaluated the entrapment efficiency of ICG and DOX within the micelles using centrifuge tubes with $100 \mathrm{kDa}$ membrane filters. $95.0 \% \pm 0.8 \%$ ICG and $82.3 \% \pm 0.8 \%$ DOX were entrapped within the micelles at the total drug loading level of $40 \%$, exhibiting a superior drug loading capacity when compared with those of the existing nanoparticles [12, $20,22,24]$. Most of the existing biodegradable polymers exhibit a relatively low drug loading capacity for the encapsulation of ICG [20-22, 24], This high loading might result from the hierarchical arrangement of amphiphilic ICG at the interfaces between micellar 
cores and aqueous phase. Possibly, the micellar cores with alkyl chains might perfectly assemble with the hydrophobic segment of surfactant-like ICG, in which its hydrophilic groups face the aqueous phase (Scheme 1/Figure A).

The aqueous stability of ICG is a prerequisite for cancer therapy, and thus we evaluated the aqueous stability of ICG in I/D-Micelles at $25^{\circ} \mathrm{C}$. Fig. 2A and Supplementary Material: Fig. S5 show that I/D-Micelles resulted in good stability of ICG in the absorbance and fluorescence at $\mathrm{pH} 4.5$ and $\mathrm{pH} 7.4$ for at least $48 \mathrm{~h}$ as compared to free ICG and DOX (ICG/DOX), indicating that the micelles can maintain a stable micellar structure in aqueous solution at acidic and physiological $\mathrm{pH}$ owing to the perfect encapsulation of ICG and DOX within the micelles [22, $34,35]$. It also implies that the micelles are able to protect double bonds in the hydrophobic segment of ICG from the degradation in acidic endocytic compartments at tumor owing to the hierarchical assembly of ICG within micellar cores.

Figure I. Synthetic route of mPEG-b-PAsp(DA)
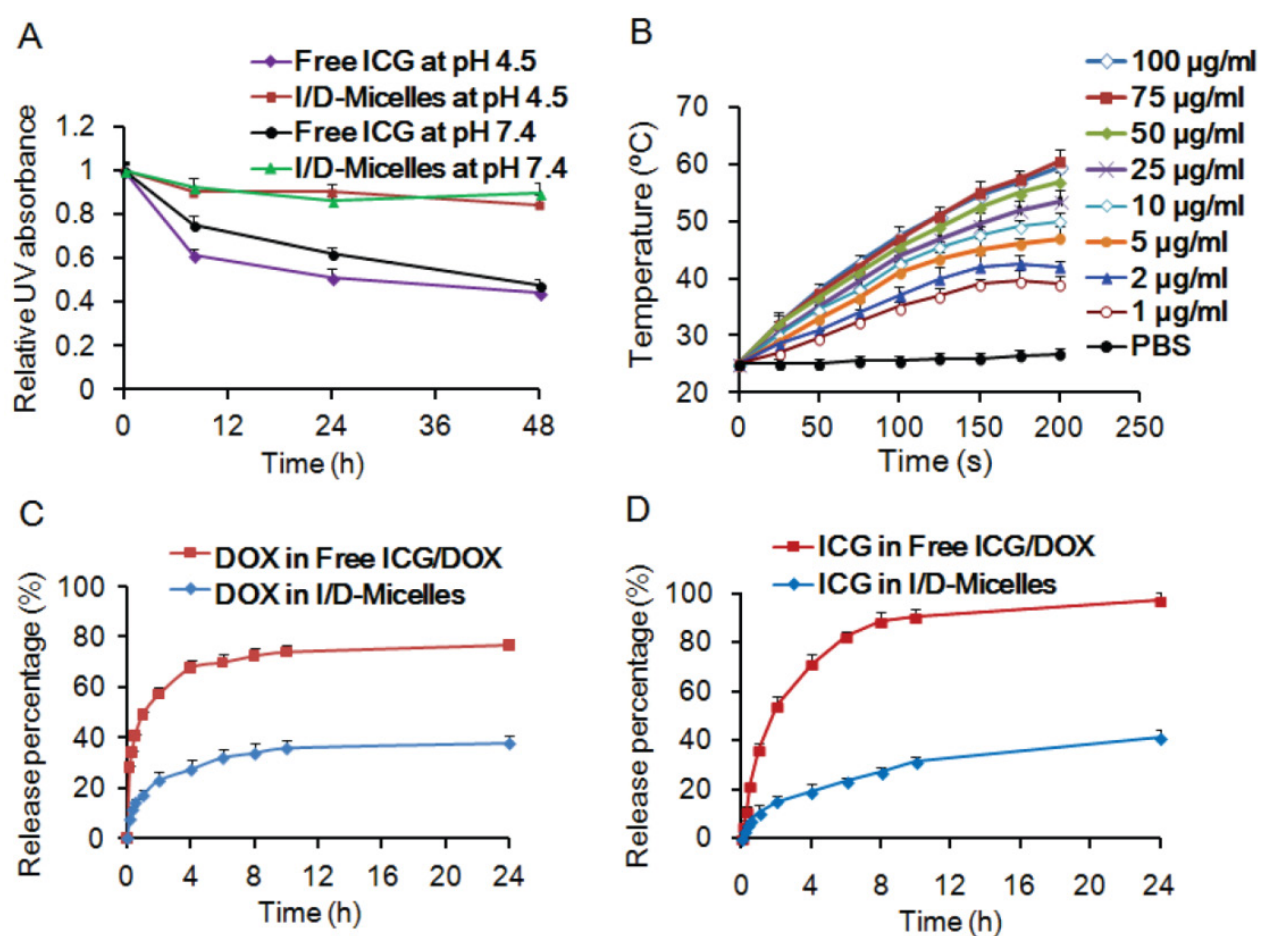

Figure 2. (A) Relative UV absorbance of ICG at $785 \mathrm{~nm}$ in aqueous solution at $\mathrm{pH} 4.5$ and 7.4 during $48 \mathrm{~h}$, (B) change of temperature of $0.5 \mathrm{~mL}$ I/D-Micelles at various concentrations of ICG as the function of photoirradiation time $\left(1.5 \mathrm{~W} / \mathrm{cm}^{2}\right)$, (C) release behavior of DOX from free ICG/DOX and I/D-Micelles in aqueous solution at $\mathrm{pH} 7.4,(\mathrm{D})$ release behavior of ICG from free ICG/DOX and I/D-Micelles in aqueous solution at $\mathrm{pH}$ 7.4. 
To demonstrate the photothermal capacity of I/D-Micelles, we also evaluated their photothermal effect at various concentrations of ICG in aqueous solution. I/D-Micelles $(0.5 \mathrm{~mL})$ exhibited a quick increase of temperature from $25{ }^{\circ} \mathrm{C}$ to $42{ }^{\circ} \mathrm{C}$ in $200 \mathrm{~s}$ at the concentration of $2.0 \mu \mathrm{g} / \mathrm{mL}$ ICG upon $1.5 \mathrm{~W} / \mathrm{cm}^{2}$ photoirradiation (Fig. 2B). PBS as a negative control did not trigger the increase of temperature. It indicates that I/D-Micelles at a relatively low concentration of ICG can quickly trigger hyperthermia during a short photoirradiation and higher concentrations can cause more significant hyperthermia, which plays a key prerequisite to trigger cell death (above $42^{\circ} \mathrm{C}$ ) [36, 37]. Additionally, I/D-Micelles also display superior photothermal capacity over free ICG/DOX, possibly owing to better stability of ICG in micelles (Supplementary Material: Fig. S6) [38, 39]. Additionally, the encapsulated ICG within micellar cores might also trigger higher condensed concentrations as compared to free ICG, and thus result in higher energy efficiency and lower heat dissipation after irradiation, which finally contribute to the enhanced photothermal effect as well. Furthermore, we demonstrated the release behaviors of ICG and DOX from I/D-Micelles at $\mathrm{pH}$ 7.4 and $37^{\circ} \mathrm{C}$. The micelles resulted in sustained release of ICG and DOX as compared to free ICG/DOX (Fig. 2C and 2D), indicating that I/D-Micelles are advantageous to minimize undesirable release for achieving targeting effect.

\section{Cellular uptake, endocytic pathways, and photothermal damage}

To demonstrate whether I/D-Micelles can be internalized by cancer cells, we evaluated their cellular uptake capability on A549 cells. Fig. 3A shows that I/D-Micelles significantly improved the cellular uptakes of ICG and DOX by 3 and 4 times after $6 \mathrm{~h}$ incubation compared to free ICG/DOX respectively, and $24 \mathrm{~h}$ incubation resulted in more significant 8-fold and 11-fold increases, indicating that the micelles can easily improve the cellular uptake of ICG and DOX [40]. The enhanced uptake can contribute to the intracellular accumulation of ICG, which is expected to reach $2.0 \mu \mathrm{g} / \mathrm{mL}$ for triggering the hyperthermia. Subsequently, the endocytic pathway of I/D-Micelles was further elucidated using flow cytometry, and several inhibitors of endocytosis including chlorpromazine, filipin, and amiloride were used to inhibit various endocytic pathways [41]. Fig. 3B shows that chlorpromazine at $10 \mu \mathrm{g} / \mathrm{mL}$ as an inhibitor of clathrin-mediated endocytosis resulted in the decrease of $60 \%$ in the cellular uptake of DOX (Supplementary Material: Fig. S7), suggesting that I/D-Micelles were mainly internalized via a clathrin-mediated endocytic pathway, which plays a key role for the internalization of the micelles into cancer cells [41]. In addition, low temperature $\left(4^{\circ} \mathrm{C}\right)$ also triggered a significant decrease of cellular uptake of DOX, indicating that the endocytotic cellular uptake is also energy-dependent [42]. The clathrin-mediated pathway allows the micelles to undergo the endolysosomal transport for their intracellular delivery [40].

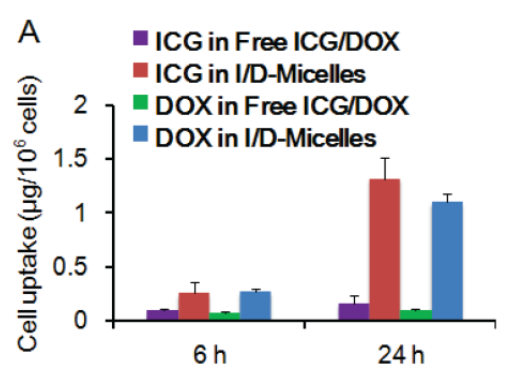

C

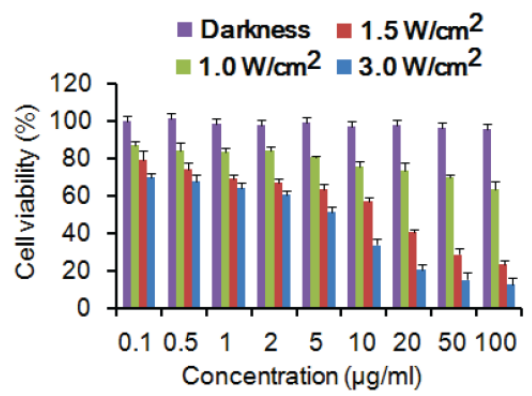

$\mathrm{B}$
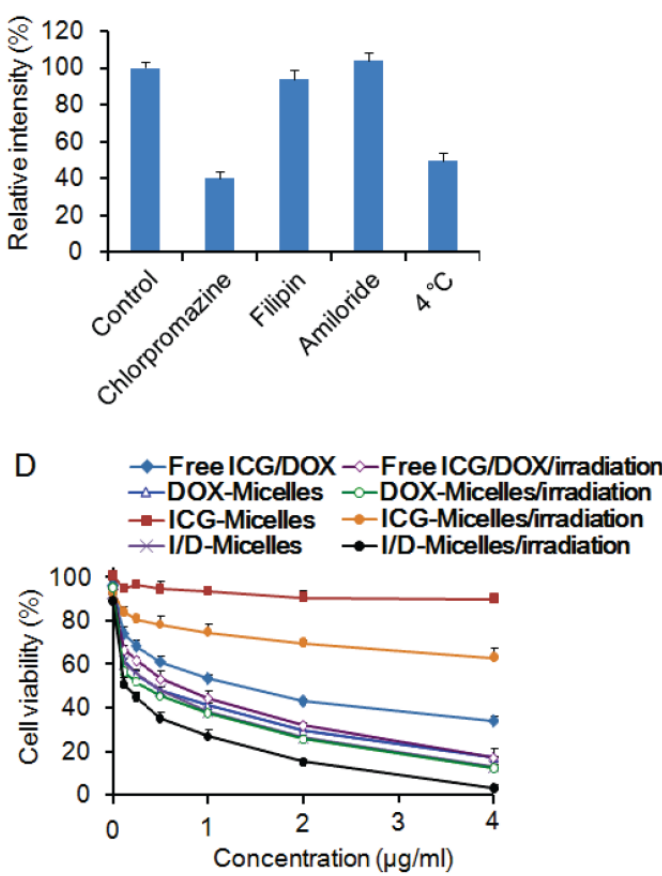

Figure 3. (A) Amounts of internalized ICG and DOX by A549 cells after $6 \mathrm{~h}$ and $24 \mathrm{~h}$ incubation with free ICG/DOX and I/D-Micelles containing $4 \mu \mathrm{g} / \mathrm{mL}$ ICG/DOX, (B) relative fluorescent intensity of I/D-Micelles internalized by A549 cells treated with PBS (control), chlorpromazine $(10 \mu \mathrm{g} / \mathrm{mL})$, filipin $(5 \mu \mathrm{g} / \mathrm{mL})$, amiloride (100 $\mu \mathrm{g} / \mathrm{mL}$ ) at $37^{\circ} \mathrm{C}$, and PBS at $4^{\circ} \mathrm{C}$ using in vitro flow cytometry analysis, (C) cell viability of A549 cells treated with 1/D-Micelles at various concentrations under darkness, 1.0 $\mathrm{W} / \mathrm{cm}^{2}, \quad 1.5 \mathrm{~W} / \mathrm{cm}^{2}$, and $3 \mathrm{~W} / \mathrm{cm}^{2}$ photoirradiation ( $3 \mathrm{~min})$, respectively, (D) cell viability of A549 cells treated with various formulations including free ICG/DOX, ICG-Micelles, DOX-Micelles and I/D-Micelles at various concentrations without or with $3 \mathrm{~min}$ photoirradiation (I.5 $\left.\mathrm{W} / \mathrm{cm}^{2}\right)$, respectively. 
To examine the photothermal injury of I/D-Micelles on cancer cells, we incubated A549 cells with the micelles encapsulating ICG alone (ICG-Micelles) in the absence of DOX for $24 \mathrm{~h}$, followed by $3 \mathrm{~min}$ photoirradiation. ICG-Micelles exhibited negligible cytotoxicity in the absence of photoirradiation and could be considered as a non-toxic agent (Fig. 3C). However, ICG-Micelles directly triggered severe photothermal damage on A549 cells upon photoirradiation $\left(\sim 12 \mu \mathrm{g} / \mathrm{mL} \quad \mathrm{IC}_{50}\right.$ at 1.5 $\left.\mathrm{W} / \mathrm{cm}^{2}\right)$, which is also dependent on the irradiation intensity and concentrations of ICG (Fig. 3C) [12, 21]. It implies that I/D-Micelles can potentially trigger severe photothermal injury on cancer cells via the enhanced cellular uptake of ICG and subsequent destabilization of subcellular structure.

To further demonstrate the synergistic cytotoxicity of I/D-Micelles between photothermal effect from ICG and chemotherapy from DOX on cancer cells, we incubated A549 cells with various formulations for 24 $\mathrm{h}$, followed by $3 \mathrm{~min}$ photoirradiation. ICG-Micelles exhibited a slight cytotoxicity against A549 cells upon photoirradiation at the relatively low concentration range $(<4 \mu \mathrm{g} / \mathrm{mL})$ (Fig. 3D). Both the micelles encapsulating DOX alone (DOX-Micelles) and I/D-Micelles without photoirradiation had the $\mathrm{IC}_{50}$ value of about $0.5 \mu \mathrm{g} / \mathrm{mL}$, indicating that the chemotherapy exhibits more obvious cytotoxicity at the similar low dose range. Importantly, I/D-Micelles displayed the $\mathrm{IC}_{50}$ value of $0.12 \mu \mathrm{g} / \mathrm{mL}$ upon photoirradiation, and possessed significant cytotoxic advantages over I/D-Micelles without photoirradiation, DOX-Micelles and free ICG/DOX [43]. The lower $\mathrm{IC}_{50}$ value is highly potential to improve the therapeutic index of anticancer agent owing to the enhanced susceptibility of cancer cells to the micelles. The cell death of I/D-Micelles was further validated by trypan blue staining of the nuclei in dead cells (Fig. 4), indicating that the cells suffered from severe damage from I/D-Micelles at a relatively low dose range [12, 43]. Obviously, I/D-Micelles exhibit synergistic cytotoxi-

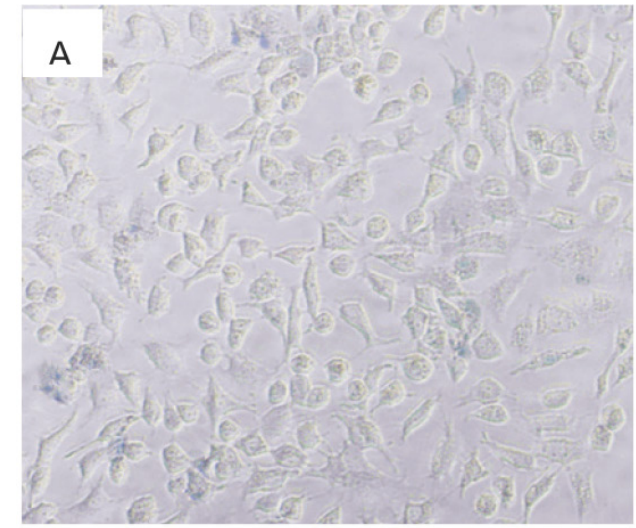

Figure 4. (A) Trypan blue staining image of A549 cells incubated with PBS under $3 \mathrm{~min}$ photoirradiation $\left(1.5 \mathrm{~W} / \mathrm{cm}^{2}\right)$, (B) trypan blue staining image of A549 cells incubated with I/D-Micelles at the concentrations of $4 \mu \mathrm{g} / \mathrm{mL}$ ICG/DOX with $3 \mathrm{~min}$ photoirradiation $\left(1.5 \mathrm{~W} / \mathrm{cm}^{2}\right)$. city via a combinational photothermo-chemotherapy. However, I/D-Micelles at a low concentration of ICG $(<2 \mu \mathrm{g} / \mathrm{mL})$ have a weak hyperthermia as Fig. 2B, so the synergistic cytotoxicity of I/D-Micelles is unlikely originated from hyperthermia in the dose range of $0.005 \sim 2 \mu \mathrm{g} / \mathrm{mL}$. Consequently, it is highly necessary to explore the key synergistic mechanism of I/D-Micelles other than photothermal therapy or chemotherapy. We hypothesize that the synergistic cytotoxicity is correlated with the generation of intracellular singlet oxygen from amphiphilic ICG [24, 44-46], which can trigger the disruption of lysosomal membranes via a photochemical internalization (PCI) effect, and subsequently induce enhanced susceptibility of cancer cells to DOX [44-46].

\section{Generation of singlet oxygen}

To validate the generation of singlet oxygen from ICG via photodynamic effect, we firstly monitored the generation of singlet oxygen in aqueous solution from I/D-Micelles using 1, 3-diphenyliso-benzofuran (DPBF) as a probe, which can specifically be quenched in the presence of singlet oxygen. Fig. 5A shows that I/D-Micelles began to generate detectable amount of singlet oxygen at a concentration of as low as $0.1 \mu \mathrm{g} / \mathrm{mL}$ upon photoirradiation, and exhibited a concentration-dependent production of singlet oxygen in aqueous solution, indicating that ICG is highly capable of inducing singlet oxygen at a low concentration. Additionally, the generation of singlet oxygen from I/D-Micelles was similar to that from free ICG/DOX (Fig. 5A). ICG exhibited negligible quenching of singlet oxygen, even though ICG was encapsulated within the micelles at a high drug loading. Possibly, amphiphilic ICG hierarchically arranges at the interface of micelles, and is able to directly release singlet oxygen into aqueous solution without significant quenching as illustrated in Fig. 5B. This hierarchical structure of ICG within micelles might be considered as a singlet oxygen-permeable structure.

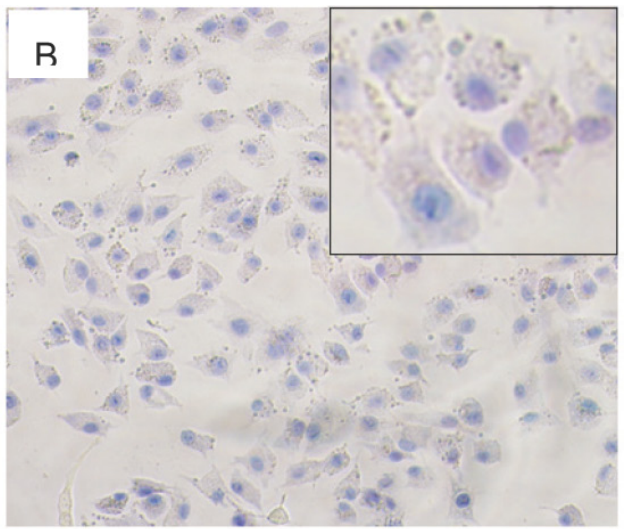



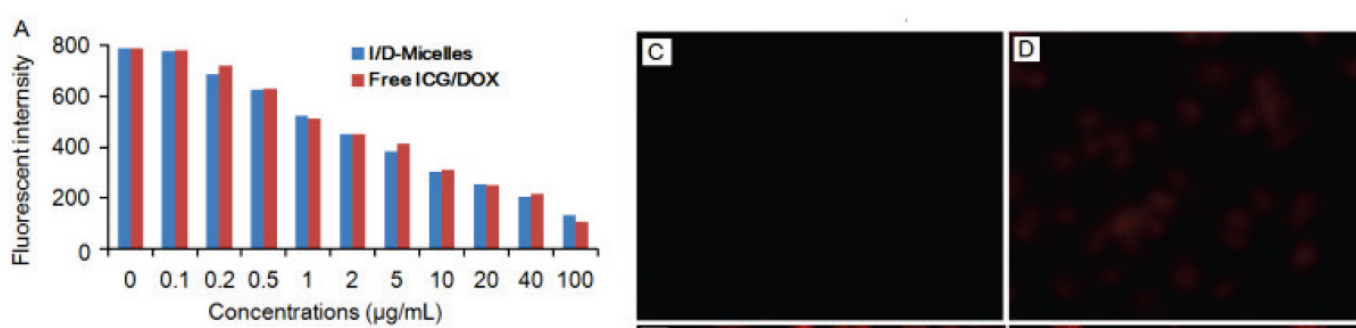

B
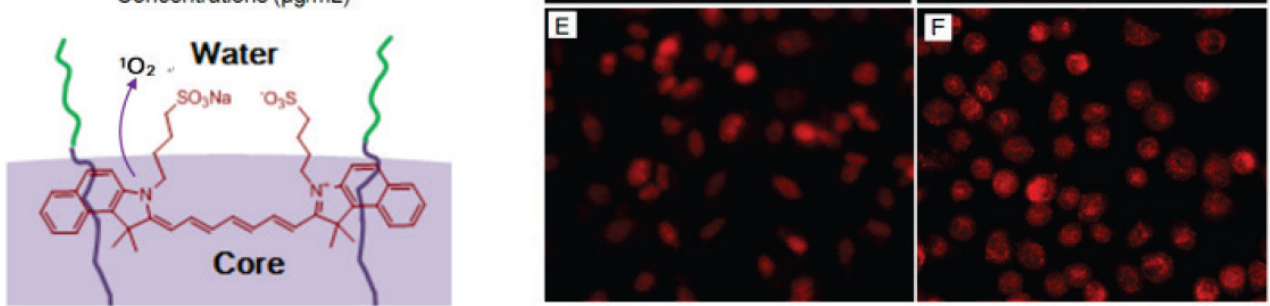

Figure 5. Fluorescent intensity of DPBF after trapping singlet oxygen from I/D-Micelles and free ICG/DOX at various concentrations of ICG under 3 min photoirradiation $\left(1.5 \mathrm{~W} / \mathrm{cm}^{2}\right)(\mathrm{A})$, proposed hierarchical arrangement of ICG at the interfaces of micelles (B), and fluorescent images of A549 cells treated with I/D-Micelles using DHE staining for singlet oxygen detection in absence of photoirradiation at the dose of $0.1 \mu \mathrm{g} / \mathrm{mL}$ ICG (C), I/D-Micelles under $3 \mathrm{~min}$ photoirradiation $\left(1.5 \mathrm{~W} / \mathrm{cm}^{2}\right)$ at the dose of $0.1 \mu \mathrm{g} / \mathrm{mL}$ ICG (D), $0.2 \mu \mathrm{g} / \mathrm{mL}$ ICG (E), and $0.5 \mu \mathrm{g} / \mathrm{mL}$ ICG (F).

To further validate the formation of intracellular singlet oxygen from I/D-Micelles, we observed the production of singlet oxygen in cells using dihydroethidium (DHE) as an intracellular reactive oxygen species (ROS) probe, which can emit red fluorescence signals inside cell nuclei after it is oxidized into 2-hydroxyehtidium by ROS [11, 47]. I/D-Micelles without photoirradiation as control group had no fluorescence, implying that I/D-Micelles cannot produce singlet oxygen in cells in the absence of photoirradiation (Fig. 5C). However, I/D-Micelles at the dose of $0.1 \mu \mathrm{g} / \mathrm{mL}$ ICG resulted in weak fuzzy fluorescence upon photoirradation (Fig. 5D), indicating that only a small amount of singlet oxygen were generated in cells at a low dose of ICG. Subsequently, red fluorescence was dramatically increased at higher doses of ICG (Fig. 5E and 5F), implying that more singlet oxygen were generated in the presence of the higher dose of ICG. It indicates that intracellular singlet oxygen was effectively generated and also exhibited a concentration-dependent increase in the dose range of $0.1 \sim 0.5 \mu \mathrm{g} / \mathrm{mL}$ ICG, in which ICG is unable to cause hyperthermia. The generation of intracellular singlet oxygen was also validated by slight photocytotoxicity of ICG-Micelles at the low dose range (Fig. 3D). Obviously, the formation of intracellular singlet oxygen from I/D-Micelles at a low dose is essential in mediating the disruption of lysosomal membranes.

We further monitored the disruption of lysosomal membranes triggered by singlet oxygen from I/D-Micelles upon photoirradiation using acridine orange (AO) as an intracellular indicator, which can emit red fluorescence in intact acidic lysosomes, and display green fluorescence in neutralized cytosol and nuclei. The confocal laser scanning microscopy imaging shows that the acidic lysosomes in A549 cells treated with PBS displayed red fluorescence in the absence of photoirradiation, which was similar to those suffered from photoirradiation (Fig. 6A and 6B). It indicates that the lysosomal compartments were still stable in the absence of ICG regardless of photoirradiation. However, the red fluorescence from AO was remarkably decreased in the presence of I/D-Micelles containing $0.1 \mu \mathrm{g} / \mathrm{mL}$ ICG upon photoirradiation, and mostly disappeared when the dose of ICG reached $0.2 \mu \mathrm{g} / \mathrm{mL}$ (Fig. 6C $\sim 6 \mathrm{~F}$ ). It indicates that I/D-Micelles at a low dose of ICG can effectively disrupt lysosomal membranes upon photoirradiation. Even though a few studies have reported that gold nanorods-mediated hyperthermia could trigger disruption of lysosomal membranes under strong irradiation [5], I/D-Micelles at a low dose range is not possible to trigger hyperthermia. Therefore, intracellular singlet oxygen from ICG possibly acts as a key role for the disruption of lysosomal membranes and thereof cytoplasmic delivery via PCI effect. Obvious$l y$, the disruption of lysosomes is highly advantageous to realize endolysosomal escape of I/D-Micelles into cytosol and potentially improve the accessibility of DOX to nucleus, which contribute to the synergistic cytotoxicity of I/D-Micelles.

\section{Biodistribution and in vivo NIRF imaging}

To demonstrate the biodistribution behavior of I/D-Micelles, we intravenously injected I/D-Micelles into the mice bearing A549 tumor and evaluated the concentrations of DOX and ex vivo NIRF intensities of ICG at various tissues at $24 \mathrm{~h}$ post-injection. Both ICG and DOX from I/D-Micelles were mainly distributed into tumor, liver, and kidneys at $24 \mathrm{~h}$ post-injection (Fig. 7A 7B). I/D-Micelles resulted in 2.5-fold and 2.4-fold higher accumulations of ICG and DOX at 
tumors compared to free ICG/DOX respectively, which might be attributed to EPR effect of the micelles with small size [24, 29]. The enhanced accumulation of ICG at tumor is able to improve signal to noise ratio of imaging (Fig. 7C), which is a key prerequisite for improving imaging contrast. Additionally, Free ICG also exhibited a slight ability to accumulate at tumor site at this dose due to its amphiphilicity as shown in Fig. 7C. On the other hand, the enhanced accumulation of DOX at tumor and its lower distribution at heart might potentially achieve superior chemotherapy with lower adverse side effect as well.

To further demonstrate the capacity of I/D-Micelles for cancer imaging, we subsequently evaluated the in vivo NIRF imaging of I/D-Micelles on the mice bearing A549 tumors. I/D-Micelles were mainly distributed into liver, kidney, gastrointestinal tract and tumor at $6 \mathrm{~h}$ post-injection (data not shown), and exhibited a significant accumulation of NIRF signals at tumor at $24 \mathrm{~h}$ post-injection (Fig. 7D), which is in accordance with its biodistribution as shown in Fig. 4B. In particular, I/D-Micelles exhibited a relatively long-term retention of detectable NIRF signals at tumor during the imaging period of $6 \mathrm{~d}$ as compared to free ICG (Supplementary Material: Fig. S8), accompanying with low noise from normal tissues such as liver, kidney and spleen after $24 \mathrm{~h}$ post-injection. Possibly, the enhanced accumulation of ICG at tumor without quick leakage and its quick elimination at normal tissues contribute to high SNR level at tumor [3, 17]. Obviously, I/D-Micelles possess good targeting capacity to tumor, which might allow us to detect the tumor at a high SNR level and achieve the good tumor localization for guiding subsequent photothermal treatment.
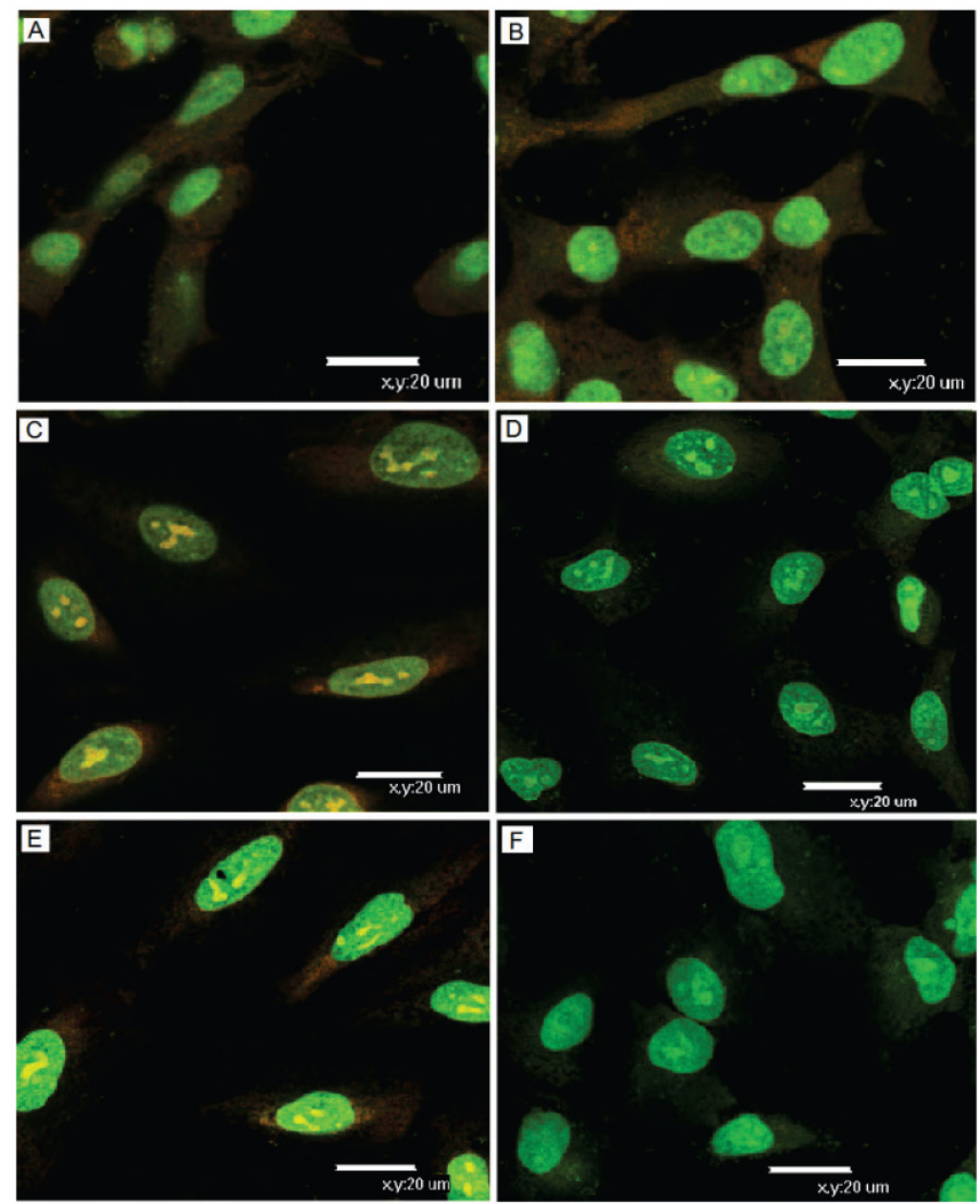

Figure 6. Observation of lysosomal disruption of $A 549$ cells treated with I/D-Micelles with 3 min photoirradiation (I.5 W/cm $\left.{ }^{2}\right)$ using AO staining. The cells were respectively treated with PBS without photoirradiation (A), PBS with photoirradiation (B), I/D-Micelles containing $0.1 \mu g / \mathrm{mL} I C G$ without photoirradiation (C), I/D-Micelles containing $0.1 \mu \mathrm{g} / \mathrm{mL}$ ICG with photoirradiation (D), I/D-Micelles containing $0.2 \mu \mathrm{g} / \mathrm{mL}$ ICG without photoirradiation (E), and I/D-Micelles containing 0.2 $\mu \mathrm{g} / \mathrm{mL}$ ICG with photoirradiation (F), followed by $\mathrm{AO}$ staining. AO as an intracellular indicator emits red fluorescence in acidic lysosomes (6I0 640 nm, red), and displays green fluorescence in neutralized cytosol and nuclei (5I5 545 nm, green). 

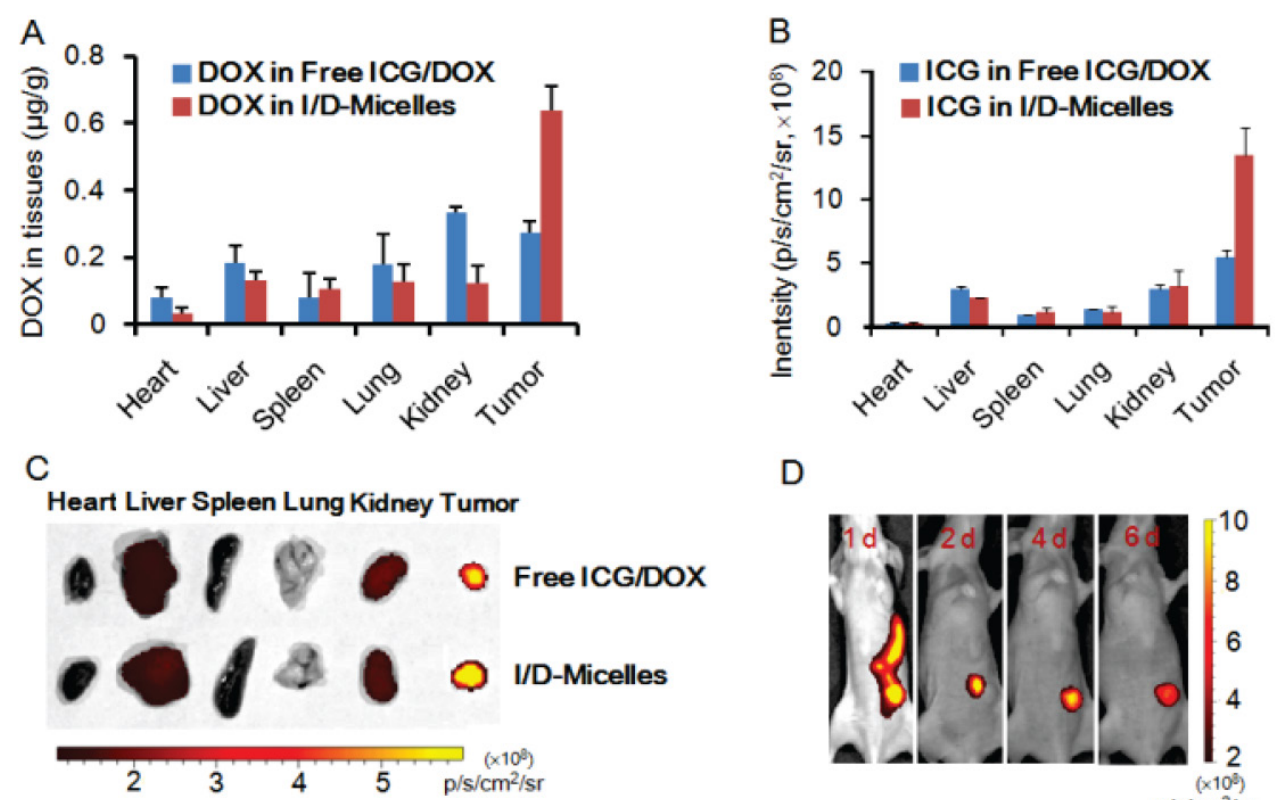

D
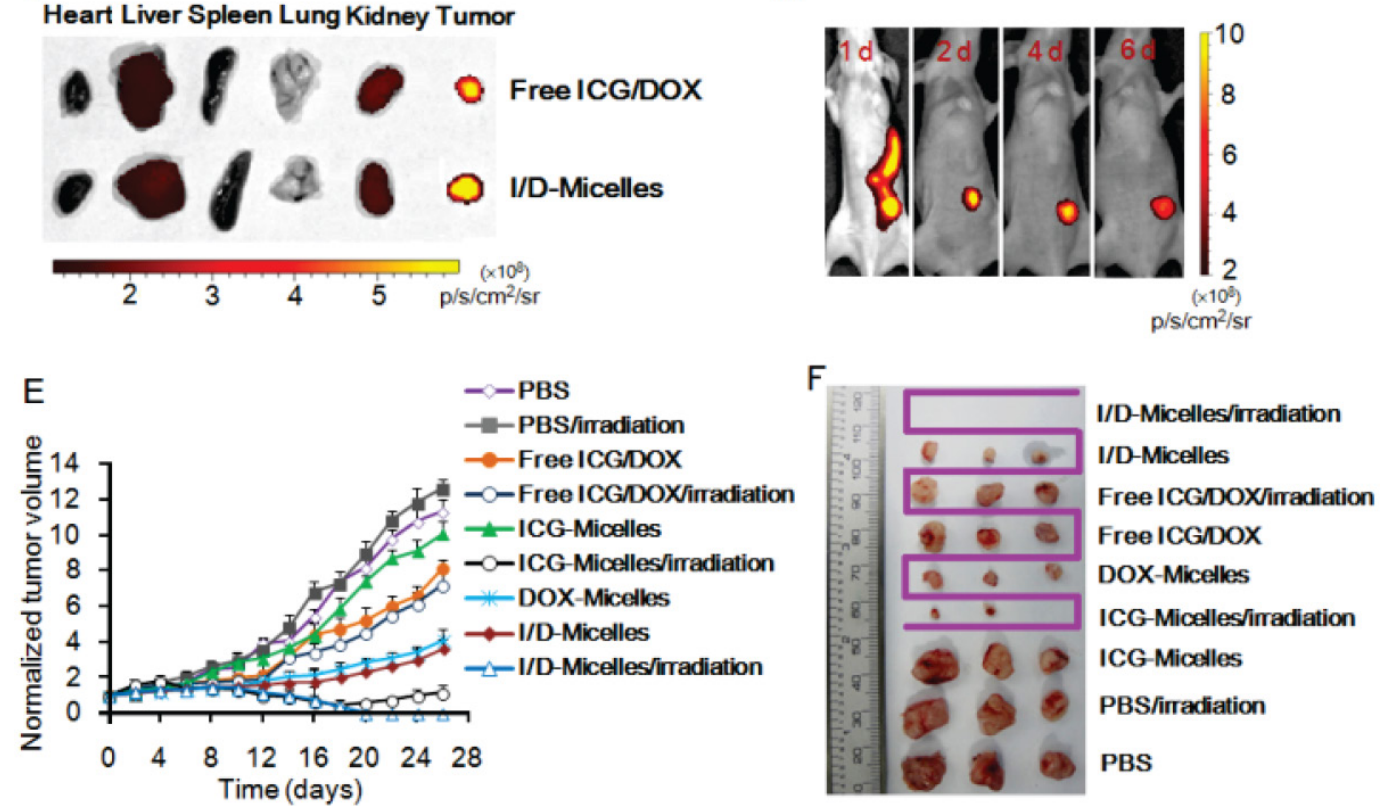

Figure 7. (A) Amounts of DOX from free ICG/DOX and I/D-Micelles in heart, liver, spleen, lung, kidney, and tumor of the mice at $24 \mathrm{~h}$ post-injection at the dose of $7.5 \mathrm{mg} / \mathrm{kg}$ ICG/DOX, respectively, (B) NIRF intensities and (C) ex vivo imaging of ICG from free ICG/DOX and I/D-Micelles in heart, liver, spleen, lung, kidney, and tumor of the mice at $24 \mathrm{~h}$ post-injection at the dose of $7.5 \mathrm{mg} / \mathrm{kg}$ ICG/DOX, respectively, (D) in vivo NIRF imaging of the mice bearing A549 tumor injected with I/D-Micelles at the dose of $7.5 \mathrm{mg} / \mathrm{kg}$ ICG/DOX at I, 2, 4 and 6 day post-injection, respectively, (E) tumor growth inhibition profiles of the mice bearing A549 tumor injected with various formulations including PBS, free ICG/DOX, ICG-Micelles, DOX-Micelles, and I/D-Micelles at the dose of 7.5 mg/kg ICG on day 0, 2, and 4, followed by $5 \mathrm{~min}$ photoirradiation $\left(1.0 \mathrm{~W} / \mathrm{cm}^{2}\right)$ or not at $24 \mathrm{~h}$ post-injection, respectively $(\mathrm{n}=3)$, $(\mathrm{F})$ photos of the tumors extracted from the mice bearing A549 tumor at the end of the experiment (I/D-Micelles exhibited completely tumor eradication and ICG-Micelles displayed tumor re-growth, $\mathrm{n}=3$ ).

\section{In vivo synergistic efficacy}

To elucidate the in vivo synergistic efficacy of theranostic micelles, I/D-Micelles were injected into the mice bearing A549 tumors at the dose of 7.5 $\mathrm{mg} / \mathrm{kg}$ ICG/DOX, and then the tumors were irradiated at $1.0 \mathrm{~W} / \mathrm{cm}^{2}$ for $5 \mathrm{~min}$ at $24 \mathrm{~h}$ post-injection. Subsequently, tumor volumes were measured for up to 26 days. The tumor volumes were normalized against their original volumes ( 0 day) for monitoring the tumor growth behavior (Fig. 7E). The control group with or without photoirradiation exhibited significant increase of tumor volumes by 10 13 times compared to their original volumes (Supplementary Material: Fig. S9), indicating that photoirradiation itself has a negligible influence on tumor growth in the absence of ICG and DOX. DOX-Micelles and I/D-Micelles without photoirradiation exhibited a similar inhibition effect on tumor growth, and their tumor volumes were increased by 3 4 times after 26 days post-injection. Obviously, DOX in I/D-Micelles plays a key role for their anticancer efficacy, and ICG exhibits no therapeutic activity in the absence of photoirradiation. Even though DOX within I/D-Micelles or DOX-micelles was effectively delivered into tumors and achieved the enhanced chemotherapy compared to free ICG/DOX, the tumors suffering from chemotherapy alone still displayed the slow tumor growth without total eradication, indicating that some cancer cells survive from the chemotherapy. On the other hand, ICG-Micelles without photoirradiation also displayed a similar tumor growth behavior to that of control group and could be considered as a nontoxic agent. Interestingly, ICG-Micelles exhibited a significant photothermal injury on tumors upon photoirradiation, and triggered the necrosis and regress of tumors $4 \sim 6$ day 
post-injection. It indicates that the micelles effectively deliver sufficient amount of ICG into the tumor and thus induce severe hyperthermia, accompanying with the generation of singlet oxygen upon photoirradiation, which jointly trigger more lethal damage on cancer cells than the cytotoxicity of DOX-Micelles or I/D-Micelles without photoirradiation (Fig. 7F) [17]. But we subsequently found the obvious tumor re-growth during 14 18 days post-injection, indicating that ICG-Micelles could not completely eradicate the tumors possibly owing to the proliferation of residual cells surviving from severe photothermal damage (Supplementary Material: Fig. S9) [17]. The residual cells in tumor possibly fail to internalize sufficient ICG for achieving hyperthermia, and singlet oxygen from ICG at a low dose only triggered a slight photodynamic damage on these cells as shown in Fig. 3D. Most importantly, I/D-Micelles caused the necrosis and regression of tumors $4 \sim 6$ day post-injection upon photoirradiation, and simultaneously achieved complete tumor eradication without any re-growth after 16 days post-injection (Supplementary Material: Fig. S9), which is superior to those of ICG-Micelles with photoirradiation, DOX-Micelles and I/D-Micelles without photoirradiation $(\mathrm{P}<0.01)$ (Fig. 7F). I/D-Micelles without photoirradiation exhibited obvious residual tumor and subsequent re-growth, implying that the residual cells do not respond to the lethal dose of DOX due to their insufficient cellular uptake of the micelles. Because DOX and ICG in I/D-Micelles are co-delivered into tumor via the endocytosis of the micelles, we can deduce that the residual tumor surviving from chemotherapy might also have insufficient cellular uptake of ICG, which is difficult to trigger its lethal photothermal injury on the residual cells. Consequently, the synergistic efficacy of I/D-Micelles in the presence of photoirradiation possibly ascribes to the synergistic cyototxicity of I/D-Micelles with the $\mathrm{IC}_{50}$ value of 0.12 $\mu \mathrm{g} / \mathrm{mL}$ triggered by singlet oxygen-mediated PCI effect at a low dose of ICG instead of photothermal injury of ICG on the residual cells. Singlet oxygen-mediated disruption of lysosomal membranes provides an effective pathway to transport more DOX to cytosol and nucleus, which can improve the susceptibility of residual cancer cells to DOX, and finally achieve the eradication of residual tumor. Generally, conventional strategies to combine two anticancer drugs or combine drug with other regimens such as radiation are frequently employed to improve anticancer efficacy. However, these strategies may trigger additional severe adverse side effects or resistance. As described in Fig. 8, the synergistic effect between cyanine dye and therapeutic agent is effective to achieve superior anticancer efficacy and can be considered as an emerging strategy to eradicate tumor without additional concerns of safety or resistance [11].

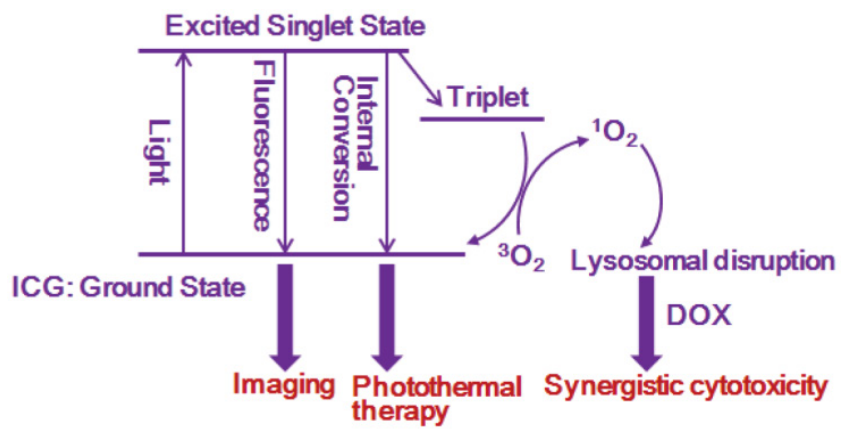

Figure 8. Mechanism illustration of cyanine-based micelles as a multimodal platform for synergistic cancer therapy.

\section{Conclusions}

In summary, we have developed a hierarchical micelle system for multimodal synergistic therapy guided by NIRF imaging. The micelle structure with alkyl chains can allow amphiphilic cyanine dye and anticancer drug to be assembled at a high drug loading, and result in the enhanced cellular uptake and tumor accumulation. I/D-Micelles can directly trigger severe photothermal damage on tumor upon photoirradiation. Simultaneously, the hierarchical assembly of cyanine dye at micelles can directly release singlet oxygen into aqueous environment, and then singlet oxygen-mediated disruption of lysosomal membranes is highly capable of triggering the synergistic cytotoxicity of anticancer drug with PTT for achieving tumor eradication without any tumor re-growth. More interestingly, the singlet oxygen-mediated synergistic mechanism can act as a popular strategy in multimodal therapy which is potentially able to synergize other therapeutic modalities such as photodynamic therapy for superior anticancer therapy.

\section{Supplementary Material}

Figs. S1 - S9. http:/ /www.thno.org/v04p0399s1.pdf

\section{Acknowledgements}

This work was supported by National Natural Science Foundation of China (81202472, 51273165), National Basic Research Program (2012CB932500, 2013CB733802), and the priority academic program development of Jiangsu higher education institutions (PAPD).

\section{Competing Interests}

The authors have declared that no competing interest exists. 


\section{References}

1. Zha Z, Yue X, Ren Q, Dai Z. Uniform polypyrrole nanoparticles with high photothermal conversion efficiency for photothermal ablation of cancer cells. Adv Mater 2013; 25: 777-82.

2. Lal S, Clare SE, Halas NJ. Nanoshell-enabled photothermal cancer therapy: impending clinical impact. Acc Chem Res 2008; 41: 1842-51.

3. Luo S, Zhang E, Su Y, Cheng T, Shi C. A review of NIR dyes in cancer targeting and imaging. Biomaterials 2011;32: 7127-38.

4. Wang S, Huang P, Nie L, Xing R, Liu D, Wang Z, et al. Single continuous wave laser induced photodynamic/plasmonic photothermal therapy using photosensitizer-functionalized gold nanostars. Adv Mater 2013; 25: 3055-61.

5. Zhang Z, Wang L, Wang J, Jiang X, Li X, Hu Z, et al. Mesoporous silica-coated gold nanorods as a light-mediated multifunctional theranostic platform for cancer treatment. Adv Mater 2012; 24: 1418-23.

6. Yang K, Zhang S, Zhang G, Sun X, Lee ST, Liu Z. Graphene in mice: ultrahigh in vivo tumor uptake and efficient photothermal therapy. Nano Lett 2010; 10: 3318-23.

7. Zheng M, Yue C, Ma Y, Gong P, Zhao P, Zheng C, et al. Single-step assembly of DOX/ICG loaded lipid--polymer nanoparticles for highly effective chemo-photothermal combination therapy. ACS Nano 2013; 7: 2056-67.

8. Xiao Z, Ji C, Shi J, Pridgen EM, Frieder J, Wu J, et al. DNA self-assembly of targeted near-infrared-responsive gold nanoparticles for cancer thermo-chemotherapy. Angew Chem Int Ed 2012; 51: 11853-7.

9. Peng J, Zhao L, Zhu X, Sun Y, Feng W, Gao Y, et al. Hollow silica nanoparticles loaded with hydrophobic phthalocyanine for near-infrared photodynamic and photothermal combination therapy. Biomaterials 2013; 34: 7905-12.

10. Peng CL, Shih YH, Lee PC, Hsieh TM, Luo TY, Shieh MJ. Multimodal image-guided photothermal therapy mediated by 188Re-labeled micelles containing a cyanine-type photosensitizer. ACS Nano 2011; 5: 5594-607.

11. Huang G, Chen HB, Luo XQ, Yu HJ, Moore Z, Bey EA, et al. Superparamagnetic iron oxide nanoparticles: amplifying ROS stress to improve anticancer drug efficacy. Theranostics 2013; 3: 116-26.

12. Yu J, Javier D, Yaseen MA, Nitin N, Richards-Kortum R, Anvari B, et al Self-assembly synthesis, tumor cell targeting, and photothermal capabilities of antibody-coated indocyanine green nanocapsules. J Am Chem Soc 2010; 132: 1929-38.

13. Barth BM, E IA, Shanmugavelandy SS, Kaiser JM, Crespo-Gonzalez D, DiVittore NA, et al. Targeted indocyanine-green-loaded calcium phosphosilicate nanoparticles for in vivo photodynamic therapy of leukemia. ACS Nano 2011; 5: 5325-37.

14. Patel RH, Wadajkar AS, Patel NL, Kavuri VC, Nguyen KT, Liu H. Multifunctionality of indocyanine green-loaded biodegradable nanoparticles for enhanced optical imaging and hyperthermia intervention of cancer. J Biomed Opt 2012; 17: 046003

15. Kassab K. Photophysical and photosensitizing properties of selected cyanines. J Photochem Photobiol B 2002; 68: 15-22.

16. Baumler W, Abels C, Karrer S, Weiss T, Messmann H, Landthaler M, et al. Photo-oxidative killing of human colonic cancer cells using indocyanine green and infrared light. Br J Cancer 1999; 80: 360-3.

17. Yang H, Mao H, Wan Z, Zhu A, Guo M, Li Y, et al. Micelles assembled with carbocyanine dyes for theranostic near-infrared fluorescent cancer imaging and photothermal therapy. Biomaterials 2013; 34: 9124-33.

18. Trachootham D, Alexandre J, Huang P. Targeting cancer cells by ROS-mediated mechanisms: a radical therapeutic approach? Nat Rev Drug Discov 2009; 8: 579-91.

19. Anraku Y, Kishimura A, Oba M, Yamasaki Y, Kataoka K. Spontaneous formation of nanosized unilamellar polyion complex vesicles with tunable size and properties. J Am Chem Soc 2010; 132: 1631-6.

20. Miki K, Kimura A, Oride K, Kuramochi Y, Matsuoka H, Harada H, et al. High-contrast fluorescence imaging of tumors in vivo using nanoparticles of amphiphilic brush-like copolymers produced by ROMP. Angew Chem Int Ed 2011; 50: 6567-70.

21. Zheng $X$, Xing $D, Z$ hou $F, W u ~ B, C h e n ~ W R$. Indocyanine green-containing nanostructure as near infrared dual-functional targeting probes for optical imaging and photothermal therapy. Mol Pharm 2011; 8: 447-56.

22. Kirchherr AK, Briel A, Mader K. Stabilization of indocyanine green by encapsulation within micellar systems. Mol Pharm 2009; 6: 480-91.

23. Escobedo JO, Rusin O, Lim S, Strongin RM. NIR dyes for bioimaging applications. Curr Opin Chem Biol 2010; 14: 64-70.

24. Altinoglu EI, Russin TJ, Kaiser JM, Barth BM, Eklund PC, Kester M, et al. Near-infrared emitting fluorophore-doped calcium phosphate nanoparticles for in vivo imaging of human breast cancer. ACS Nano 2008; 2: 2075-84.

25. Lovell JF, Jin CS, Huynh E, Jin H, Kim C, Rubinstein JL, et al. Porphysome nanovesicles generated by porphyrin bilayers for use as multimodal biophotonic contrast agents. Nat Mater 2011; 10: 324-32.

26. Liu TW, Chen J, Burgess L, Cao W, Shi J, Wilson BC, et al. Multimodal bacteriochlorophyll theranostic agent. Theranostics 2011; 1: 354-62.

27. Lovell JF, Liu TW, Chen J, Zheng G. Activatable photosensitizers for imaging and therapy. Chem Rev 2010; 110: 2839-57.

28. Murakami M, Cabral H, Matsumoto $\mathrm{Y}, \mathrm{Wu} \mathrm{S}$, Kano MR, Yamori T, et al. Improving drug potency and efficacy by nanocarrier-mediated subcellular targeting. Sci Trans Med 2011; 3: 64ra2.
29. Cabral H, Matsumoto $\mathrm{Y}$, Mizuno $\mathrm{K}$, Chen Q, Murakami M, Kimura M, et al. Accumulation of sub-100 $\mathrm{nm}$ polymeric micelles in poorly permeable tumours depends on size. Nat Nanotechnol 2011; 6: 815-23.

30. Hirsjarvi S, Passirani C, Benoit JP. Passive and active tumour targeting with nanocarriers. Curr Drug Discov Technol 2011; 8: 188-96.

31. Matsumura Y. Preclinical and clinical studies of NK012, an SN-38-incorporating polymeric micelles, which is designed based on EPR effect. Adv Drug Deliv Rev 2011; 63: 184-92.

32. Matsumura Y. Poly (amino acid) micelle nanocarriers in preclinical and clinical studies. Adv Drug Deliv Rev 2008; 60: 899-914.

33. Anraku Y, Kishimura A, Kobayashi A, Oba M, Kataoka K. Size-controlled long-circulating PICsome as ruler to measure critical cut-off disposition size into normal and tumor tissues. Chem Commun 2011; 47: 6054-6

34. Gao $Y$, Chen $Y$, Ji X, He X, Yin $\mathrm{Q}$, Zhang Z, et al. Controlled intracellular release of doxorubicin in multidrug-resistant cancer cells by tuning the shell-pore sizes of mesoporous silica nanoparticles. ACS Nano 2011; 5: 9788-98.

35. Babin J, Pelletier M, Lepage M, Allard J-F, Morris D, Zhao Y. A new two-photon-sensitive block copolymer nanocarrier. Angew Chem Int Ed 2009; 48: 3329-32.

36. Jang B, Park JY, Tung CH, Kim IH, Choi Y. Gold nanorod-photosensitizer complex for near-infrared fluorescence imaging and photodynamic/photothermal therapy in vivo. ACS Nano 2011; 5: 1086-94.

37. You J, Zhang G, Li C. Exceptionally high payload of doxorubicin in hollow gold nanospheres for near-infrared light-triggered drug release. ACS Nano 2010; 4: 1033-41.

38. Ke H, Wang J, Dai Z, Jin Y, Qu E, Xing Z, et al. Gold-nanoshelled microcapsules: a theranostic agent for ultrasound contrast imaging and photothermal therapy. Angew Chem Int Ed 2011; 50: 3017-21.

39. Robinson JT, Tabakman SM, Liang Y, Wang H, Casalongue HS, Vinh D, et al. Ultrasmall reduced graphene oxide with high near-infrared absorbance for photothermal therapy. J Am Chem Soc 2011; 133: 6825-31.

40. Maxfield FR, McGraw TE. Endocytic recycling. Nat Rev Mol Cell Biol 2004; 5 : 121-32.

41. Iversen TG, Skotland T, Sandvig K. Endocytosis and intracellular transport of nanoparticles: Present knowledge and need for future studies. Nano Today 2011; 6: 176-85.

42. Wang F, Wang YC, Dou S, Xiong MH, Sun TM, Wang J. Doxorubicin-tethered responsive gold nanoparticles facilitate intracellular drug delivery for overcoming multidrug resistance in cancer cells. ACS Nano 2011; 5: 3679-92.

43. Huang P, Lin J, Wang X, Wang Z, Zhang C, He M, et al. Light-triggered theranostics based on photosensitizer-conjugated carbon dots for simultaneous enhanced-fluorescence imaging and photodynamic therapy. Adv Mater 2012; 24: 5104-10.

44. Castano AP, Demidova TN, Hamblin TR. Mechanisms in photodynamictherapy: part one-photosensitizers, photochemistry and cellular localization. Photodiagnosis Photodyn Ther 2004; 1: 279-93.

45. Nishiyama $\mathrm{N}$, Iriyama $\mathrm{A}$, Jang WD, Miyata $\mathrm{K}$, Itaka $\mathrm{K}$, Inoue $\mathrm{Y}$, et al. Light-induced gene transfer from packaged DNA enveloped in a dendrimeric photosensitizer. Nat Mater 2005; 4: 934-41.

46. Berg K, Selbo PK, Prasmickaite L, Tjelle TE, Sandvig K, Moan D, et al. Photochemical internalization: A novel technology for delivery of macromolecules into cytosol. Cancer Res 1999; 59: 1180-3.

47. He T, Banach-Latapy A, Vernis L, Dardalhon M, Chanet R, Huang ME. Peroxiredoxin 1 knockdown potentiates beta-lapachone cytotoxicity through modulation of reactive oxygen species and mitogen-activated protein kinase signals. Carcinogenesis 2013;: 1-10. 